doi: 10.29277/cardio.36.3.8

\title{
Cardiología digital (e-Cardiología): herramientas de utilidad para el diagnóstico y el manejo del paciente con fibrilación auricular
}

\author{
Dr. Walter Reyes Caorsi, FHRS*
}

\section{Resumen}

La tecnología digital móvil (mSalud), recurso aún subutilizado, tiene el potencial para transformarse en un auxiliar imprescindible en la práctica clínica, en particular en Cardiología. La fibrilación auricular es la arritmia más frecuente, cuya prevalencia aumenta con la edad y tiene como complicación más temida el accidente cerebrovascular. Su prevención depende de un diagnóstico oportuno, una adecuada estratificación de riesgo y el uso de anticoagulantes orales. Sin embargo, es con frecuencia paroxística y asintomática, lo que dificulta su diagnóstico. El aporte de la tecnología mediante el desarrollo de aplicaciones para celulares, relojes, dispositivos portátiles o implantables que registran el ritmo cardíaco y permiten el monitoreo prolongado, ambulatorio y a distancia facilitan su detección. Se repasan las principales evidencias que justifican la aplicación de estas estrategias de tamizaje, cuando está indicado realizarlo, cómo y a quiénes hacerlo. Por último, se plantea también la utilidad de estas estrategias de monitoreo prolongado en el manejo y la prevención de la fibrilación auricular.

Palabras clave: SALUD MÓVIL

CARDIOLOGÍA DIGITAL

FIBRILACIÓN AURICULAR

PREVENCIÓN CARDIOVASCULAR

ACCIDENTE CEREBROVASCULAR

\section{Digital Cardiology (e-Cardiology): useful tools for diagnosis and manage- ment of atrial fibrillation}

\section{Summary}

The mobile digital technology (mHealth), a tool still underutilized, has the potential to become an essential aid in clinical practice, particularly in Cardiology. Atrial fibrillation is the most frequent arrhythmia whose prevalence increases with age and its most feared complication is cerebrovascular accident. Its prevention depends on a timely diagnosis, an adequate risk stratification and the use of oral anticoagulants. However, it is frequently paroxysmal and asymptomatic, which makes its diagnosis difficult. The contribution of technology through the development of applications for cell phones, watches, portable or implantable devices that record the heart rhythm and allow prolonged, outpatient and remote monitoring, facilitate its detection. The main evidences that justify the application of these screening strategies are reviewed, when it is indicated to perform it, how and to whom to do it. Finally, the usefulness of these prolonged monitoring strategies in the management and prevention of atrial fibrillation is also considered.

Key words: $\quad$ mHEALTH

DIGITAL CARDIOLOGY

ATRIAL FIBRILLATION

CARDIOVASCULAR PREVENTION

STROKE

Comisión Honoraria para la Salud Cardiovascular. Montevideo, Uruguay.

Correspondencia: Dr. Walter Reyes Caorsi. Correo electrónico: wreyes001@gmail.com

El autor declara no tener conflictos de interés.

Recibido Set 6, 2021; aceptado Oct 15, 2021 


\section{Cardiologia digital (e-Cardiologia): ferramentas úteis para o diagnóstico e tratamiento de pacientes com fibrilação atrial}

\section{Resumo}

A utilização da tecnologia digital móvel (m-Saúde), ainda pouco utilizada, tem potencial para se tornar um auxílio essencial na prática clínica, principalmente na Cardiologia. A fibrilação atrial é a arritmia mais frequente, cuja prevalência aumenta com a idade e sua complicação mais temida é o acidente vascular cerebral. Sua prevenção depende de um diagnóstico oportuno, de uma estratificação de risco adequada e do uso de anticoagulantes orais. Porém, freqüentemente é paroxística e assintomática, o que dificulta seu diagnóstico. A contribuição da tecnologia por meio do desenvolvimento de aplicativos para telefones celulares, relógios, dispositivos portáteis ou implantáveis que registram o ritmo cardíaco e permitem monitoramento prolongado, ambulatorial e remoto, facilitam sua deteç̧ão. São revisadas as principais evidências que justificam a aplicação dessas estratégias de rastreamento, quando é indicado fazê-lo, como e para quem fazê-lo. Finalmente, a utilidade dessas estratégias de monitoramento prolongado no manejo e prevenção da fibrilação atrial também é considerada.

Palavras chave: SAÚDE MOVIL

\section{CARDIOLOGIA DIGITAL}

FIBRILAÇÃO ATRIAL

PREVENÇÃO CARDIOVASCULAR

ACIDENTE VASCULAR CEREBRAL

Las nuevas tecnologías digitales tienen el potencial de transformar la práctica asistencial en las próximas décadas, a través del diagnóstico más rápido y confiable de las enfermedades infecciosas, el empoderamiento de los pacientes para monitorizar y manejar sus patologías crónicas, la promoción de salud y el bienestar a través de aplicaciones personalizadas y los cuidados médicos fuera de los ambientes tradicionales a través del monitoreo remoto. The Topol Review. Preparing the healthcare work force to deliver the digital future. NHS (National Health Service). Reino Unido. Febrero, 2019.

\section{Introducción}

La tecnología digital móvil y portable es utilizada habitualmente con diversos objetivos en nuestra vida cotidiana. En Medicina y en particular en Cardiología este recurso, aún subutilizado, tiene el potencial de transformarse en un auxiliar imprescindible en la valoración clínica y en el adecuado seguimiento de muchos pacientes con patologías crónicas de importancia pronóstica.

La llamada mSalud o salud móvil ha sido definida por la Organización Mundial de la Salud (OMS) como "la práctica médica y de salud pública apoyada en dispositivos móviles como teléfonos celulares, dispositivos de monitorización de pacientes, asistentes digitales personales y otros dispositivos inalámbricos"(1). El uso de los teléfonos celulares es lo que se ve con más facilidad. Los médicos utilizamos en forma cotidiana diversas aplicaciones (apps) como fuente de consultas o asesoramiento; asimismo, el uso de estos dispositivos y diversas apps se ha desarrollado de manera explosiva entre los usuarios que intentan mejorar sus cuidados de salud. Debemos tener conciencia que inevitablemente estamos en medio de una revolución digital en la práctica médica, que es crucial asumir la responsabilidad de su aplicación y desarrollo y que estos objetivos son compartidos por médicos, pacientes e industria ${ }^{(2)}$. Las principales sociedades científicas del mundo han publicado en los últimos años recomendaciones para el uso y el desarrollo de las tecnologías digitales en la asistencia médica, en general y en particular para patología cardiovascular ${ }^{(3-9)}$.

¿Cuál es la aceptación de la sociedad en cuanto al uso y preferencias de esta tecnología disponible en salud? Más allá de la información existente sobre las características de nuestro país y las opiniones de los pacientes y operadores que resumimos en anterior publicación ${ }^{(10)}$, internacionalmente y según datos de la Encuesta de consumidores realizada por el Centro Stanford en Salud Digital ${ }^{(11)}$, globalmente el uso de medios digitales en salud creció más de 10\% entre 2019 y 2020 . Este porcentaje incluye la videoconsulta, la utilización de dispositivos portátiles usables o ponibles (wearables) y el seguimiento de parámetros a distancia. Particularmente y comparando en este caso entre los años 2015 y 2020, el uso de la videoconsulta pasó de $7 \%$ a $43 \%$, el uso de dispositivos de $13 \%$ a $43 \%$ y el uso de aplicaciones para control de parámetros (pasos, actividad, etc.) de $18 \%$ a $54 \%$. No menos importante es la comprobación que $2 / 3$ de los pacientes buscan información en internet sobre su patología, síntomas o tratamientos (figura 1). En el mismo período se detecta, además del aumento significativo de la videoconsulta, una disminución de las consultas telefónicas y del contacto a través 


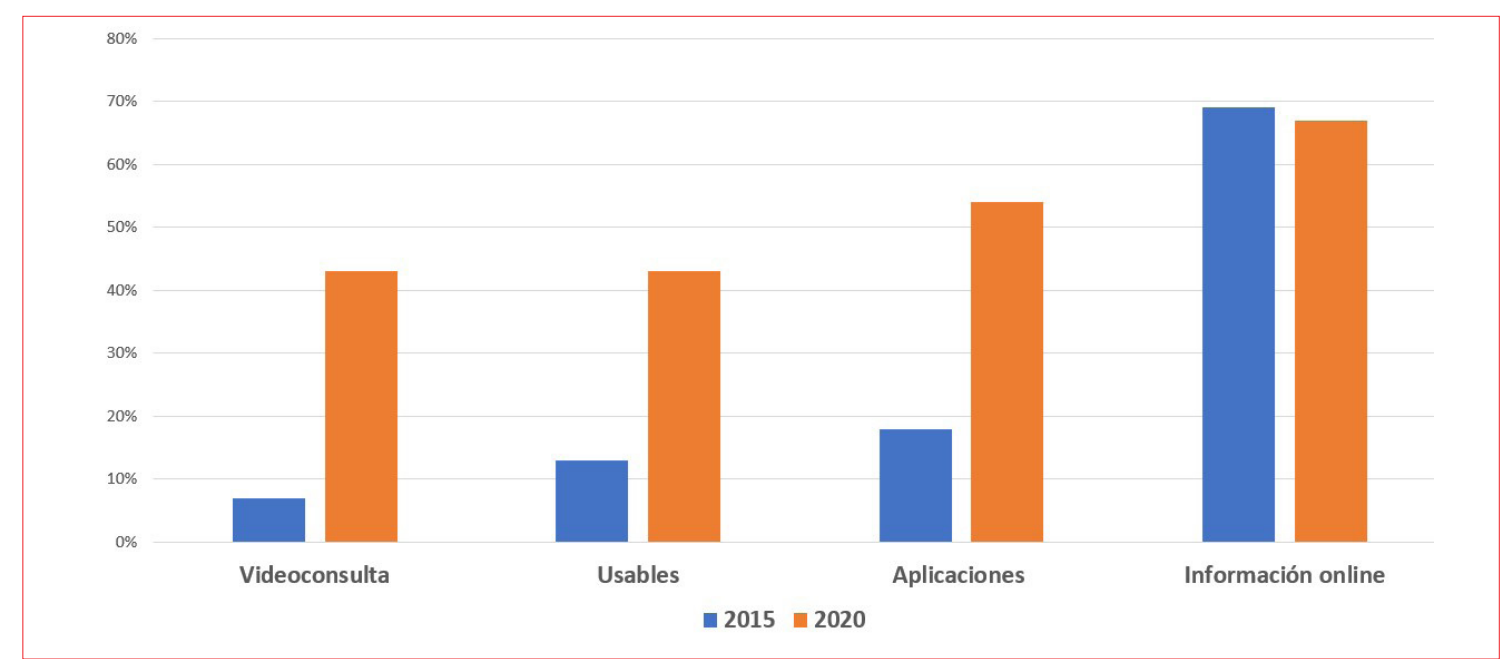

Figura 1. Adopción de herramientas digitales en salud. Modificado de referencia 11. Encuesta a consumidores sobre el uso de tecnologías en Salud Digital 2020. Stanford Center for Digital Health.

de mensajes de texto o correo electrónico. El 70\% de los consultados manifestaron el deseo de poder compartir los datos colectados en sus aplicaciones (de actividad física, control del peso, control de diabetes, por ejemplo) con sus médicos.

Otra encuesta realizada en marzo de 2020, en este caso entre médicos de la Asociación Americana del Ritmo Cardíaco (HRS por su sigla en inglés) y sus similares de Latinoamérica y Asia Pacífico (LA-HRS y AP-HRS) fue contestada online por 1.601 colegas de 77 países $^{(12)}$. El análisis de las respuestas permitió concluir que la mayoría de los médicos (62\%) recomendaban el uso de dispositivos de salud digitales para la detección de fibrilación auricular (FA), mayoritariamente el dispositivo KardiaMobile ${ }^{\circledR}$ y el Apple Watch ${ }^{\circledR}$. Reclamaban, sin embargo, una mayor facilidad para integrar estos datos a la historia clínica digital y un mayor involucramiento de las sociedades científicas para establecer normas para optimizar este recurso. Con similar metodología online, una encuesta europea de 2019 tuvo la participación de 417 médicos. Como ventajas del sistema de monitoreo fueron destacadas la facilidad de su utilización, que logra rápidamente el involucramiento del paciente, la rapidez y la eficacia en lograr un diagnóstico, su disponibilidad y la posibilidad del control a largo plazo. Se anotaron algunos inconvenientes; el principal fue la sobrecarga de datos para el análisis de los profesionales ${ }^{(13)}$.

\section{¿Por qué hacer screening de FA?}

La FA es no solo una arritmia muy frecuente, cuya prevalencia aumenta con la edad, sino que genera una importante morbimortalidad. Multiplica por 5 el riesgo de accidente cerebrovascular (ACV), su consecuencia más dramática. El mecanismo fisiopatológico es embólico y habitualmente se trata de trombos grandes que generan infartos cerebrales de gran tamaño. Por tanto, tienen mayor mortalidad y secuelas más importantes. Su importancia se jerarquiza aún más si consideramos que pueden ser recurrentes. Sin embargo, está ampliamente demostrado que son prevenibles: depende de un diagnóstico y estratificación de riesgo oportunos y del uso de anticoagulantes en quienes esté indicado. Más allá de si la FA es la causa o un marcador de riesgo de ACV, quizás la indicación de anticoagulación sea la más trascendente que podremos hacer a un paciente en quien se detecta esta arritmia. En forma alarmante se ha reportado que en Latinoamérica el 70\% de los pacientes con un ACV y FA conocida no reciben tratamiento anticoagulante. Además, el 80\% de los portadores de FA desconocen que la padecen ${ }^{(14)}$. De acuerdo con el estudio clásico de Friberg y col., la FA tiene una prevalencia de $33 \%$ en pacientes con ACV isquémico, y $10 \%$ de estos ocurren en casos con FA no diagnosticada ${ }^{(15)}$. No obstante, no es esta su única consecuencia: la FA compromete la calidad de vida, la función cardíaca, aumenta la mortalidad y recientemente se ha vinculado también a deterioro cognitivo y demencia ${ }^{(16)}$.

En un número significativo de casos la FA es intermitente y además puede ser asintomática o presentarse con síntomas inespecíficos que no orientan a una causa arrítmica. La FA asintomática ha sido asociada en forma independiente a un mayor riesgo de ACV y mortalidad compara- 


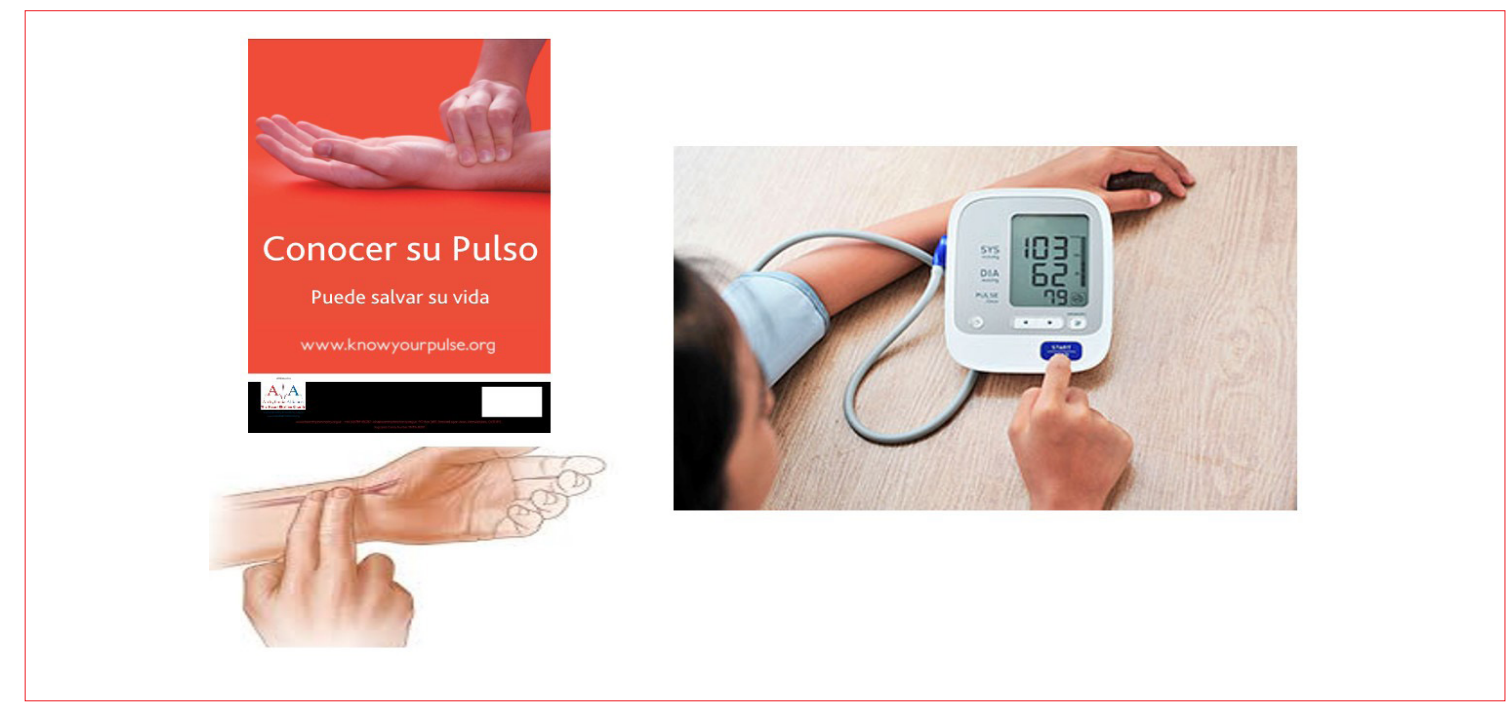

Figura 2. Estrategias para autocontrol o control oportunístico del ritmo.

Tabla 1. Estrategias de detección de fibrilación auricular. Modificado de referencias 6 y 7.

\begin{tabular}{ll}
\hline Oportunista & $\begin{array}{l}\text { Detección aprovechando } \\
\text { las oportunidades: en cada } \\
\text { consulta médica, ya sea } \\
\text { coordinada o de emergencia. }\end{array}$ \\
Sistemática & $\begin{array}{l}\text { Metodológico a todos los } \\
\text { pacientes que cumplan } \\
\text { determinadas condiciones. }\end{array}$ \\
Comunitaria & $\begin{array}{l}\text { A las personas que viven en } \\
\text { determinada área. }\end{array}$ \\
$\begin{array}{l}\text { Población de alto } \\
\text { riesgo }\end{array}$ & $\begin{array}{l}\text { Metodológico, pos-ACV, por } \\
\text { ejemplo. }\end{array}$ \\
\hline
\end{tabular}

da con la sintomática ${ }^{(6)}$. Por otra parte, estudios observacionales mostraron que la respuesta al tratamiento anticoagulante es similar a cuando la FA es detectada clínicamente. En consecuencia, su diagnóstico no es sencillo con las herramientas clínicas clásicas, examen físico y ECG, y puede pasar desapercibida ${ }^{(6)}$. Considerando su trascendencia clínica y estas dificultades diagnósticas, es necesario y recomendable utilizar estrategias adicionales para su detección ${ }^{(17)}$.

Recientemente fue presentado en el congreso anual de la Asociación Europea del Ritmo Cardíaco (EHRA 2021) y luego publicado en The Lancet el estudio STROKESTOP con el reporte del seguimiento a más de 6 años de una población en la cual se utilizó una estrategia de screening ${ }^{(18,19)}$. El objetivo no solo fue detectar FA, sino además investigar si la estrategia de monitoreo se traducía en beneficio clínico, reduciendo morbilidad y mor-
Tabla 2. Sensibilidad-especificidad de las principales estrategias clínicas y tecnológicas para detección de FA. Modificado de referencia 21.

\begin{tabular}{lccc}
\hline Método & Sensibilidad & Especificidad & Requiere ECG \\
\hline Toma del pulso & 94 & 72 & Sí \\
$\begin{array}{l}\text { Aparato de } \\
\text { presión digital }\end{array}$ & 95 & 90 & Sí \\
$\begin{array}{l}\text { Celular PPG } \\
\text { Celular }\end{array}$ & 93 & 94 & Sí \\
$\begin{array}{l}\text { inteligente } \\
\text { Reloj } \\
\text { inteligente }\end{array}$ & 98 & 97 & No \\
\hline & & 84 & No \\
\hline
\end{tabular}

talidad. Se trata de un estudio comunitario donde se invitó a participar en un protocolo de detección de FA a todas las personas de 75 y 76 años residentes en dos regiones de Suecia, sin criterios de exclusión, y fueron randomizados 1:1. Poco más de la mitad (51,3\%, 7.165/13.979) de la población aceptó. Las características clínicas de ambos grupos eran similares y 55\% fueron mujeres. En aquellos que no tenían FA conocida se realizó, mediante un dispositivo, un registro de ECG de una derivación durante $30^{\prime \prime}$ dos veces al día durante dos semanas. De detectarse FA se indicaba anticoagulación. El punto final primario combinado considerado fue ACV isquémico, embolia sistémica, sangrado grave y muerte de cualquier causa. El score $\mathrm{CHA}_{2} \mathrm{DS}_{2} \mathrm{VASc}$ fue 3,5 de promedio. El seguimiento mostró que el grupo asignado a screening tuvo un número levemente menor, estadísti- 


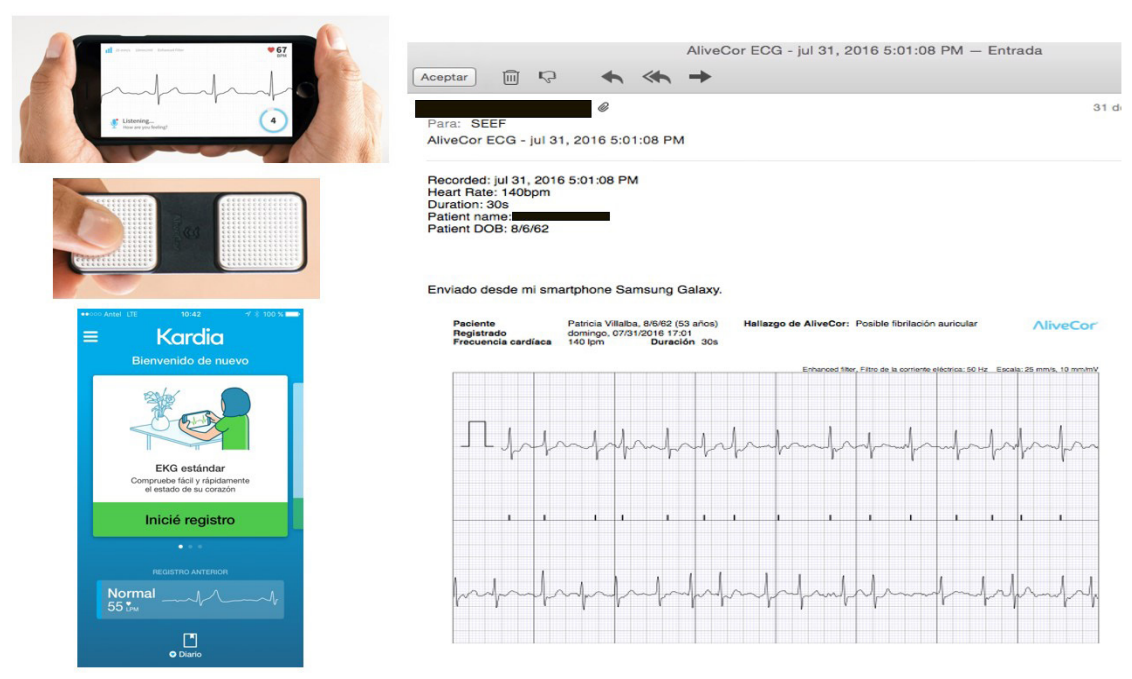

Figura 3. Sistema KardiaMobile de Alivecor®. A izquierda y de arriba abajo, celular, dispositivo y pantalla inicial de la app. A derecha, archivo pdf originado.

camente significativo de eventos respecto al grupo control (HR 0,96; 95\% CI 0,92-1,0; p =0,045). Si se analizan los resultados referidos al grupo de los realmente "tratados", se evidencia que la diferencia se basó fundamentalmente en la menor incidencia de ACV en relación con los controles $($ HR 0,76; 95\% CI 0,680,87)(18,19).

Teniendo en cuenta este contexto, sumado a las cifras epidemiológicas que permiten estimar que la prevalencia de esta arritmia se duplicará en 30 años y considerando los aspectos aún no resueltos: a quién, cómo y cuándo hacer screening, la Unión Europea ha financiado un proyecto denominado AFFECT-EU Project con el objetivo de desarrollar una estrategia de detección utilizando aplicaciones digitales de monitorización del ritmo que permitan disminuir la carga de ACV y otras comorbilidades relacionadas a la $\mathrm{FA}^{(20)}$. Los principales objetivos de este ambicioso proyecto incluyen: determinar la magnitud del problema sanitario y la relevancia de la detección de FA, la precisión de los métodos digitales, comparar las estrategias en cuanto a su rendimiento y eficacia, su viabilidad y aceptación, las implicancias económicas, etcétera.

¿Cuándo hacer tamizaje de fibrilación auricular? El objetivo principal de la detección de FA es poder identificar la población con mayor riesgo de ACV para establecer una terapia preventiva efectiva y segura, que en la mayoría de los casos implica el uso de anticoagulantes orales. Las nuevas tecnologías han simplificado la monitorización, pero el rendimiento de la estrategia a utilizar debe adap- tarse a cada situación particular. En la tabla 1 se resume la definición de las principales estrategias utilizadas extractadas de los documentos de consenso de las principales sociedades científicas del mundo ${ }^{(9)}$.

¿Cómo hacer el tamizaje de fibrilación auricular? En la pesquisa de cualquier patología, lo más importante en la selección del método a usar es su sensibilidad. Los métodos disponibles para detección de FA son múltiples, ya sea clínicos o usando tecnología y la mayoría tiene sensibilidad y especificidad muy buenas. El más sencillo es la toma del pulso buscando una irregularidad continua, aunque es el de menor especificidad. Se utiliza para detección oportunística en caso de una consulta médica o al servicio de salud, o para autocontrol enseñando la maniobra al paciente o a sus familiares; requiere siempre la realización de un ECG para confirmación de la arritmia. También pueden utilizarse para el autocontrol seriado los aparatos de control de la presión arterial digitales, que al medir la presión detectan el ritmo y alertan de su irregularidad; también requieren de confirmación con ECG (figura 2).

El desarrollo tecnológico ha puesto a disposición una gran variedad de dispositivos que facilitan la detección de $\mathrm{FA}^{(21)}$. Se ha reportado la existencia de más de cien mil apps y de más de cuatrocientos dispositivos portátiles para la monitorización de parámetros cardiovasculares ${ }^{(6)}$. Los sistemas más sencillos son apps para celulares inteligentes, muchas gratuitas, disponibles para todos los sistemas operativos. Usan fotopletismo- 


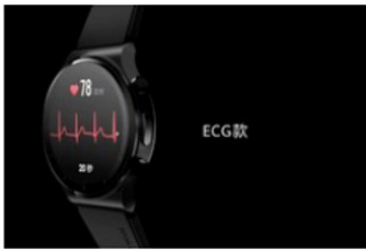

El Huawei Watch GT 2 Pro ECG

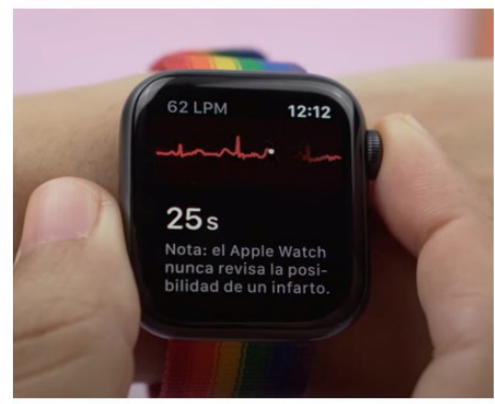

Apple Watch

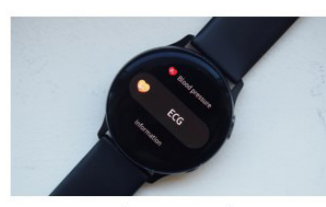

Galaxy Watch 3

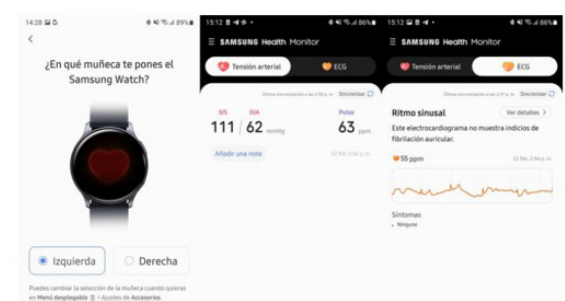

Samsung Watch

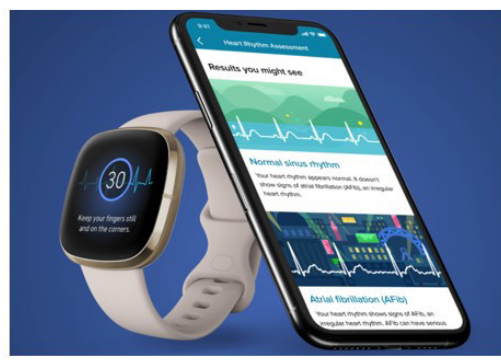

Fitbit Versa

Figura 4. Algunas de las opciones de relojes inteligentes disponibles en el mercado.

grafía (PPG), tecnología similar a la usada por los oxímetros, a través de un haz de luz y la cámara del teléfono, registran la onda de pulso pudiendo así definir su irregularidad. Son de uso muy amigable, sencillas y no hacen necesario ningún entrenamiento especial. Estos sistemas requieren, al igual que los métodos clínicos antes mencionados, la confirmación con un ECG. Aunque su sensibilidad y especificidad son buenas, sin duda los dispositivos que permiten registrar una derivación del ECG son superiores y, además, no requieren de la confirmación con un ECG completo. En las últimas guías clínicas de la Sociedad Europea de Cardiología (ESC) se establece (recomendación clase I) que el diagnóstico de FA puede hacerse en base al registro de un ECG de 12 derivaciones o un registro de $30^{\prime \prime}$ de una derivación del $\mathrm{ECG}^{(6)}$. En la tabla 2 se resumen la sensibilidad y la especificidad de estas diferentes metodologías.

Existen diferentes dispositivos (parches, relojes, lentes, cinturones) que registran una derivación del ECG, vinculados en general al celular por bluetooth ${ }^{\circledR}$, que han permitido avanzar en la precisión diagnóstica y en la frecuencia del diagnóstico de FA. Son también sencillos de usar, tienen excelente sensibilidad y especificidad y son ideales para detección en poblaciones asintomáticas. Por ejemplo, el sistema Kardia de Alivecor ${ }^{\circledR}$, de los más populares, es un dispositivo pequeño y fácilmente portable, vinculado al celular al cual puede adherirse, conectándose en forma inalámbrica a este a través de una app. Abriendo la aplicación y apoyando dos dedos sobre el dispositivo ubicado cercano o sobre el celular se registra una derivación del ECG durante 30". De manera automática, el algoritmo de la app genera un reporte que tiene tres opciones: ritmo sinusal, FA o sin clasificar. El trazado obtenido se dispone en formato pdf y puede ser archivado, compartido, etc. (figura 3).

Este sistema, por ejemplo, fue usado en el estudio REHEARSEAF ${ }^{(22)}$, en el cual se aleatorizaron 1.001 personas mayores de 65 años con un score de CHADSVASc $\geq 2$ y sin historia de FA, a registrar un ECG de 30" dos veces por semana durante un año versus los controles habituales. Se registraron 19 FA nuevas en el grupo Kardia versus 5 en grupo control (HR 3,9; 95\% IC 1,410,4; $\mathrm{p}=0,007)$.

El KardiaMobile de AliveCor ${ }^{\circledR}$ diseñado para uso individual fue utilizado en el estudio piloto realizado por la Comisión Honoraria para la Salud Cardiovascular con el objetivo de valorar su confiabilidad en el tamizaje de FA en una población de riesgo ${ }^{(23)}$. Se comparó un registro de 30" del dispositivo con un ECG de 12 derivaciones tomado en la misma instancia. En este caso se aplicó en una población del Plan Ibirapitá, incluyéndose 114 personas (78 mujeres) con una edad media de 72 años. La sensibilidad para la detección de FA fue de $100 \%$ y la especificidad 96,6\%. No hubo falsos negativos, aunque se destaca un 18,4\% de registros con diagnóstico automático de "sin clasificar" por la aplicación.

Este estudio piloto se basó en un único regis- 


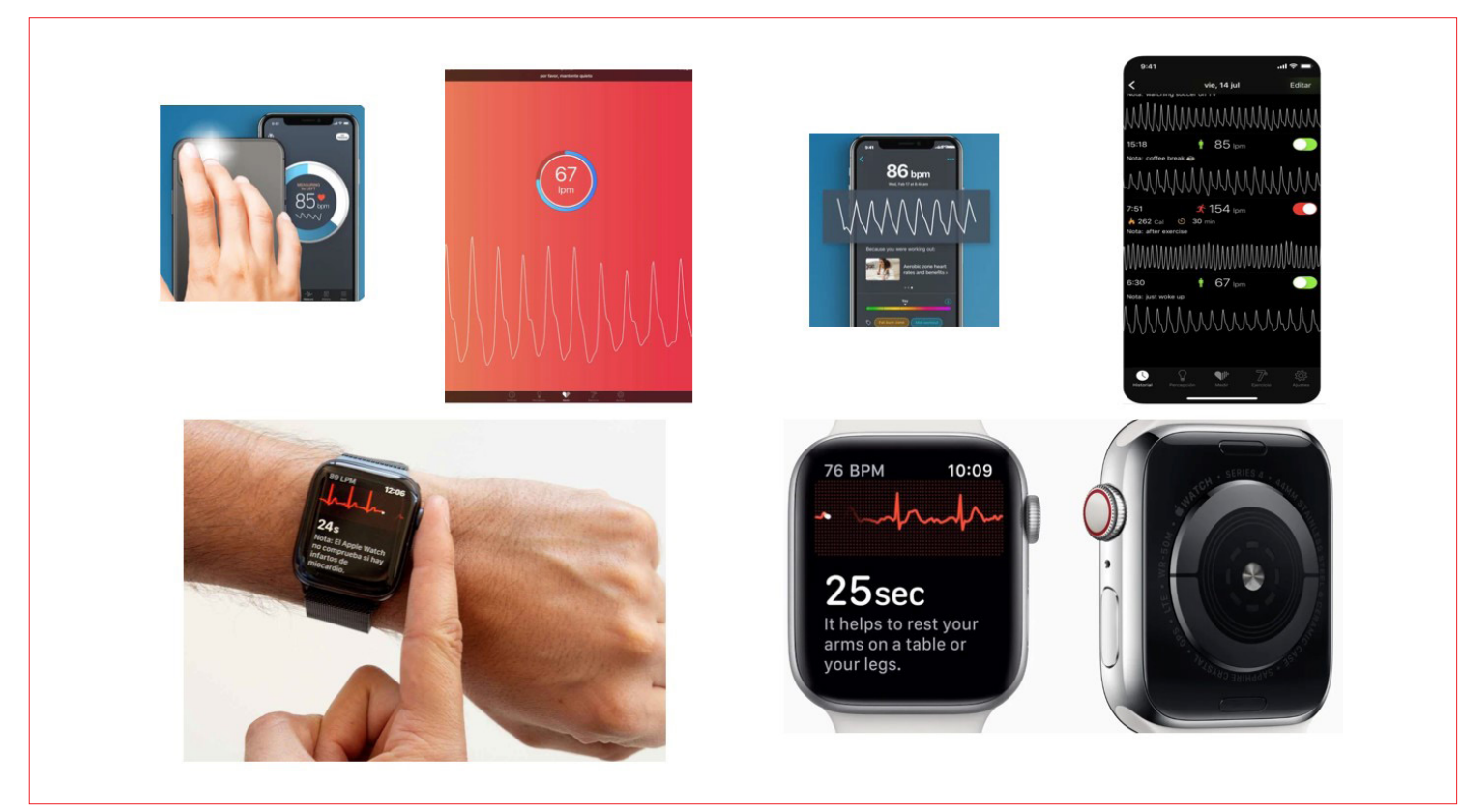

Figura 5. Arriba, app del celular usando PPG, registrando la onda de pulso. Abajo, registro con el Apple Watch ${ }^{\circledR}$.

tro de 30". Cada cuánto debería reiterarse este control en la población objetivo para optimizar su rendimiento sigue siendo una pregunta sin respuesta definitiva. En un ensayo llevado a cabo en China, usando también el dispositivo AliveCor ${ }^{\circledR}$ Heart Monitor, se incluyeron pacientes de varios centros comunitarios residentes en la provincia de Shanghái, considerando como únicos criterios de inclusión ser mayores de 65 años y no tener historia de FA. Fueron randomizados a un solo control anual o controles trimestrales (n 4.120 en cada grupo). El control trimestral se asoció a un aumento significativo en la detección de FA (HR 1,71; 95\% IC 1,062,76; $p=0,029)^{(24)}$.

Existe un gran desarrollo y competencia en diversos fabricantes de relojes inteligentes. Algunos permiten no solo el registro del ECG, una o más derivaciones durante $30^{\prime \prime}$, sino también de la presión arterial y de la saturación de $\mathrm{O}_{2}$. Varias empresas conocidas los han desarrollado: Samsung, Garmin, Huawei, Fitbit, Galaxy y otras (figura 4). El más conocido y estudiado es el Apple Watch ${ }^{\circledR}$ que usando tecnología $\mathrm{PPG}$ registra un tacograma y mide los intervalos pico a pico que interpreta como RRs. Aplicando un algoritmo propio puede determinar su regularidad. Sin embargo, la precisión de este algoritmo para definir FA puede estar comprometida por la presencia de extrasístoles o por el movimiento. Pero, además, incorporando la tecnología del sistema KardiaMobile de AliveCor ${ }^{\circledR}$, es posible registrar una derivación del ECG a través de un circuito establecido entre un detector ubicado en la parte inferior en contacto con la piel y un dedo apoyado en la perilla. Una vez registrados los 30" del ECG genera automáticamente un reporte y es almacenado en el celular vinculado desde donde puede disponerse. Es conveniente hacer el registro en reposo para una mejor definición (figura 5).

El Apple Heart Study valoró el algoritmo diagnóstico de FA utilizando $\mathrm{PPG}^{(25)}$. En este estudio participaron 419.297 personas mayores de 18 años, en los cuales se registraba un tacograma de 1 minuto cada 2 horas. Aquellos en los que se identificaba un ritmo irregular recibían por correo un parche para registrar el ECG en forma permanente durante 7 días, que se devolvería de la misma forma. El ECG obtenido por el parche era luego analizado por dos médicos. De los participantes, solo $0,52 \%$ recibieron una notificación inicial de ritmo irregular y considerando aquellos que devolvieron el parche el valor predictivo de FA de la notificación fue de $84 \%$. Si consideramos a las personas de más de 65 años, 3,2\% recibieron notificaciones. De manera similar en el Huawei Heart Study ${ }^{(26)}$, realizado en China, se usaron dos dispositivos de esa empresa, una pulsera y un reloj, que registraban el ritmo y su regularidad usando también un algoritmo basado en PPG. Se incluyeron 187.912 mayores de 18 años que se monitorizaron durante 14 días. De ellos, 424 (0,23\%) recibieron la notificación de posible FA. Entre los que se avanzó en la confirmación diagnóstica, el 87\% tenían FA. También en este estudio tanto el 


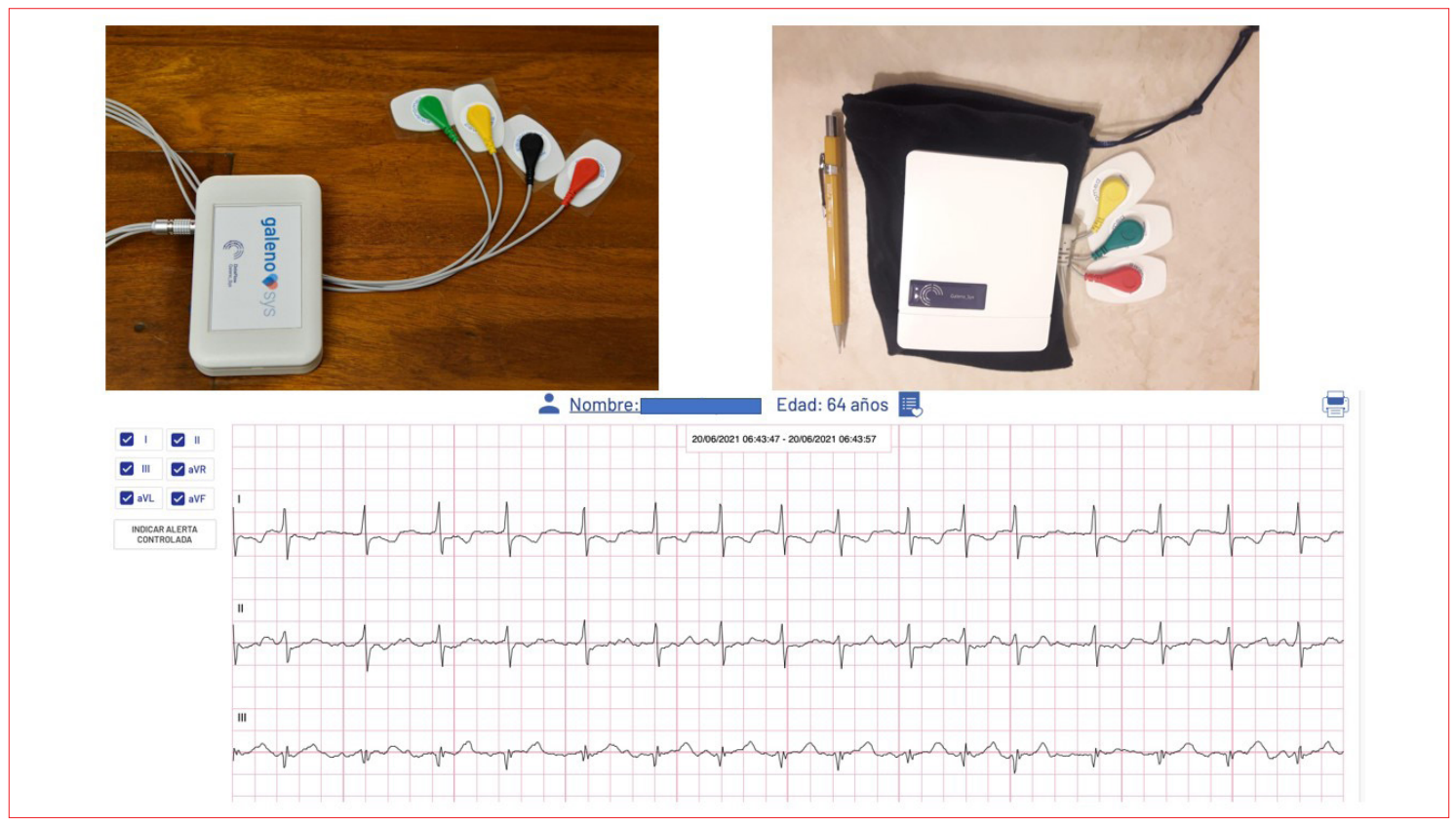

Figura 6. Sistema Galeno-Sys en desarrollo en nuestro país.

reporte de posible FA como su confirmación fue más frecuente en mayores de 65 años.

Intentando proyectarnos hacia el futuro cercano en el desarrollo de estas herramientas y considerando su avasallante avance, debemos mencionar que ya existen sistemas de monitorización del ritmo cardíaco sin contacto con la piel. Analizan las señales de PPG obtenidas de la imagen de la cara de una persona a través de la cámara del celular, detectando los cambios de coloración de la piel vinculados al ciclo cardíaco ${ }^{(27)}$. Otra línea de desarrollo tecnológico aplica un sistema de sonar similar al usado en los radares, que basado en el parlante del celular emite señales continuas en frecuencia modulada. La señal de cada latido cardíaco es recibida por el micrófono y luego de un complejo procesamiento y el uso de varios algoritmos, concluye en una señal que permite computar los intervalos RR, su variabilidad y definir la probabilidad de la existencia de $\mathrm{FA}^{(28)}$. En estudios clínicos preliminares realizados para validar la idea, se comprobó una excelente correlación entre los RR medidos de esta forma y el ECG.

Los registros reiterados multiplican por 3 la posibilidad de detectar una FA, ya sea en relación con el registro único o con estudios de Holter de 24-48 horas. De cualquier manera, son siempre controles intermitentes. Cuando se desea un monitoreo continuo la tecnología pone también a disposición otras plataformas. Por ejemplo, existe un sistema de parches descartables de 14 días de duración a prueba de agua que permiten un moni- toreo permanente durante ese período. Concluido este, el parche se retira y son leídos los registros cuyo reporte es enviado al médico referente. Es de uso más confortable para el paciente que un sistema de Holter prolongado ${ }^{(21)}$.

En nuestro país también existen iniciativas en desarrollo con sistemas de monitoreo/telemetría permanente en línea en pleno desarrollo. Se trata de un dispositivo del tamaño de una caja de cigarrillos conectado al paciente con 4 electrodos torácicos, que permite registrar en forma continua $y$ por el período de tiempo que sea necesario, hasta 6 derivaciones del ritmo cardíaco. La información es enviada en forma continua a un servidor en la nube, lo que permite analizar desde cualquier dispositivo, celular o computadora el ECG en tiempo real o el análisis histórico del registro del paciente con jerarquización de alarmas mediante la aplicación de un algoritmo. Es muy práctico para monitorización ambulatoria de largo plazo, para telemetría intrahospitalaria o en internación domiciliaria (figura 6).

Varias empresas han desarrollado registradores de eventos en asa (loop recorders) implantables en el tejido celular subcutáneo. De forma similar a los registradores de eventos externos, los implantados registran el ECG cuando son activados por el paciente, en este caso aplicando un magneto sobre el dispositivo, o cuando se activa de manera automática su algoritmo de detección. $\mathrm{Al}$ inicio fueron indicados para la evaluación de pacientes con síntomas esporádicos probablemen- 


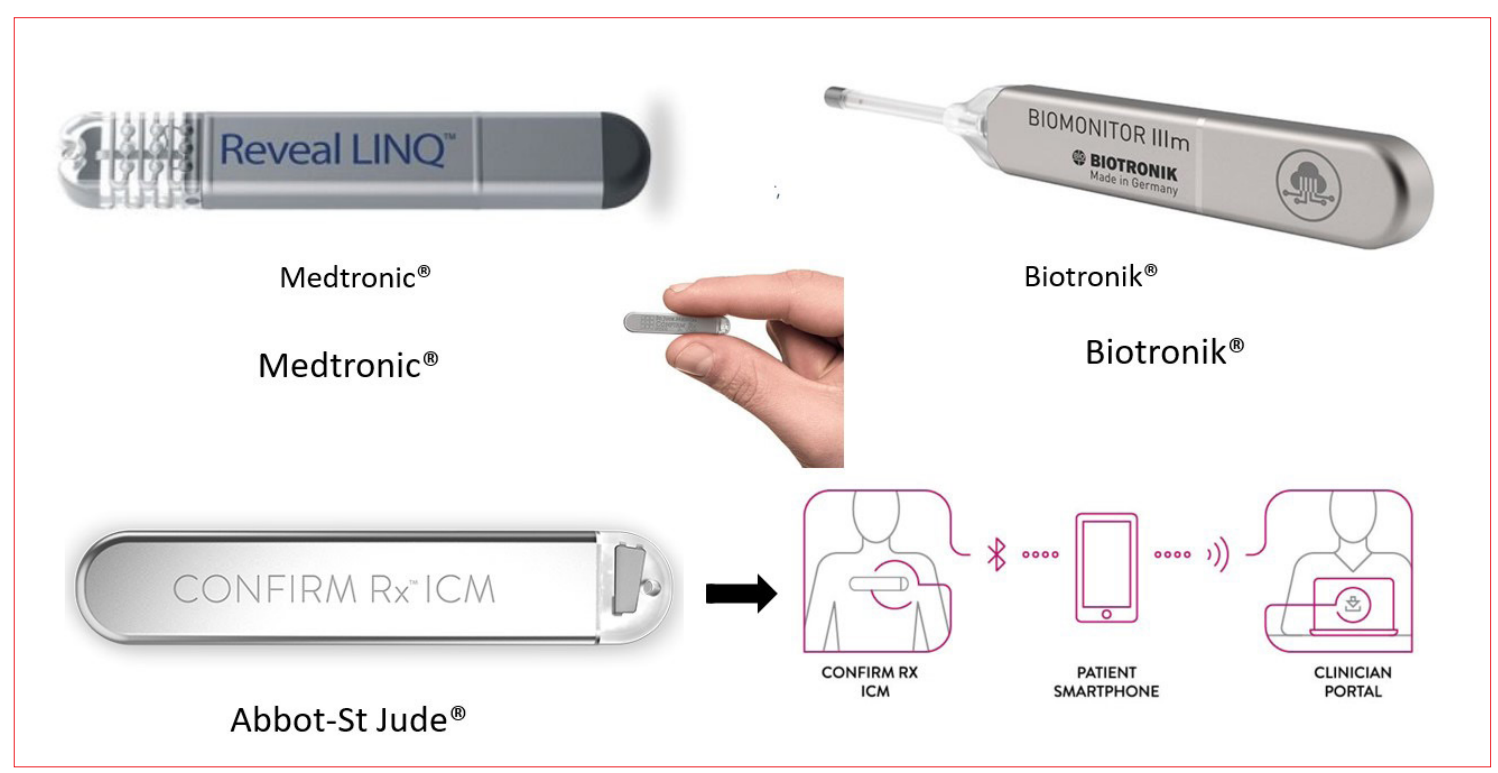

Figura 7.

te vinculados a arritmias como palpitaciones o síncope de causa no explicada. Los nuevos dispositivos tienen algoritmos específicos para la detección de FA; su batería dura varios años (figura 7). Se han utilizado básicamente para tratar de detectar FA en pacientes que han tenido un ACV. La información disponible es limitada y la incidencia reportada de nueva FA es muy variable debido a diferencias en las poblaciones estudiadas, a la tecnología de los mismos dispositivos y al tiempo de seguimiento. En la actualidad, su indicación para el diagnóstico de FA es limitada, aunque aún no conocemos reportes con los dispositivos más recientes $^{(6,8)}$.

\section{¿En quiénes pesquisar fibrilación auricular?}

La eficacia y el rendimiento del screening dependen de la población objetivo, del método utilizado en cuanto a su certeza diagnóstica y de los costos de su aplicación. Los factores de riesgo que deben considerarse para seleccionar la población a valorar son básicamente los incluidos en el score $\mathrm{CHA}_{2} \mathrm{DS}_{2} \mathrm{VASc}$ de estimación del riesgo de $\mathrm{ACV}$.

Las diferentes estrategias para detección de FA, ya sea oportunista o sistemática, han sido evaluadas en numerosos estudios involucrando diferentes poblaciones. Algunos estudios referidos a poblaciones de riesgo, otros en la comunidad o con pacientes internados o en oportunidad de concurrir a centros asistenciales. Globalmente la tasa de detección fue de 0,9\% (95\% IC, 0,7-1,1), lo cual determina que el número de personas a controlar sería de 111 por cada diagnóstico de $\mathrm{FA}^{(8)}$. Sin em-
Tabla 3. Recomendaciones para la indicación de tamizaje de FA. Modificado de referencia 4.

NO screening: < 65 años; sin factores de riesgo.

Screening OPORTUNÍSTICO (pulso-ECG):

- Apnea del sueño (clase Ila-C).

- $\geq 65$ años con hipertensión (clase I-B).

Screening SISTEMÁTICO, intermitente o prolongado:

- $\geq 75$ años o alto riesgo de ACV (clase Ila-B).

Screening SECUNDARIO, a largo plazo o dispositivos implantables:

- Pos-ACV (clase I-B/lla-B).

bargo, teniendo en cuenta la relación demostrada entre la presencia de FA y la edad, ajustando para los mayores de 65 años como lo hace un metaanálisis que involucra a más de 140.000 personas, la tasa de detección con un solo control del ritmo fue de 1,4\% (95\% IC, 1,13-1,82). En este caso, el número necesario de individuos a incluir sería de 69 por cada FA detectada ${ }^{(29)}$.

Estos resultados fueron independientes de la geografía y del método de detección utilizado, aunque se trató siempre de un registro único. El ensayo SAFE (Screening for AF in the Elderly) realizado en el Reino Unido, randomizó pacientes de 65 o más años a chequeo oportunístico versus sistemático, que se compararon con un grupo control de seguimiento habitual (5.000 incluidos por 


\begin{tabular}{|c|c|c|}
\hline Teleconsulta & Check (sintomas, frecuencia, ritmo) & Manejo integrado de la FA \\
\hline Historia clínica detallada & App a demanda. & Involucrar al paciente. \\
\hline Autorreporte de factores de riesgo & Ritmo y frecuencia usando PPG. & Equipo multidisciplinario. \\
\hline Factores de riesgo adicionales & Correlación síntomas-ritmo. & Uso de tecnología. \\
\hline $\begin{array}{l}\text { Compromiso y educación del } \\
\text { paciente }\end{array}$ & $\begin{array}{l}\text { App aprobada (CE), sensibilidad } \\
96 \% \text {, especificidad } 97 \% \text {. }\end{array}$ & $\begin{array}{l}\text { Tratamiento completo y exhaustivo: } \\
\text { control del ritmo o de la frecuencia; } \\
\text { ACO; factores de riesgo y } \\
\text { precipitantes. }\end{array}$ \\
\hline Autocontrol empoderamiento & Datos seguros en la "nube". & \\
\hline
\end{tabular}

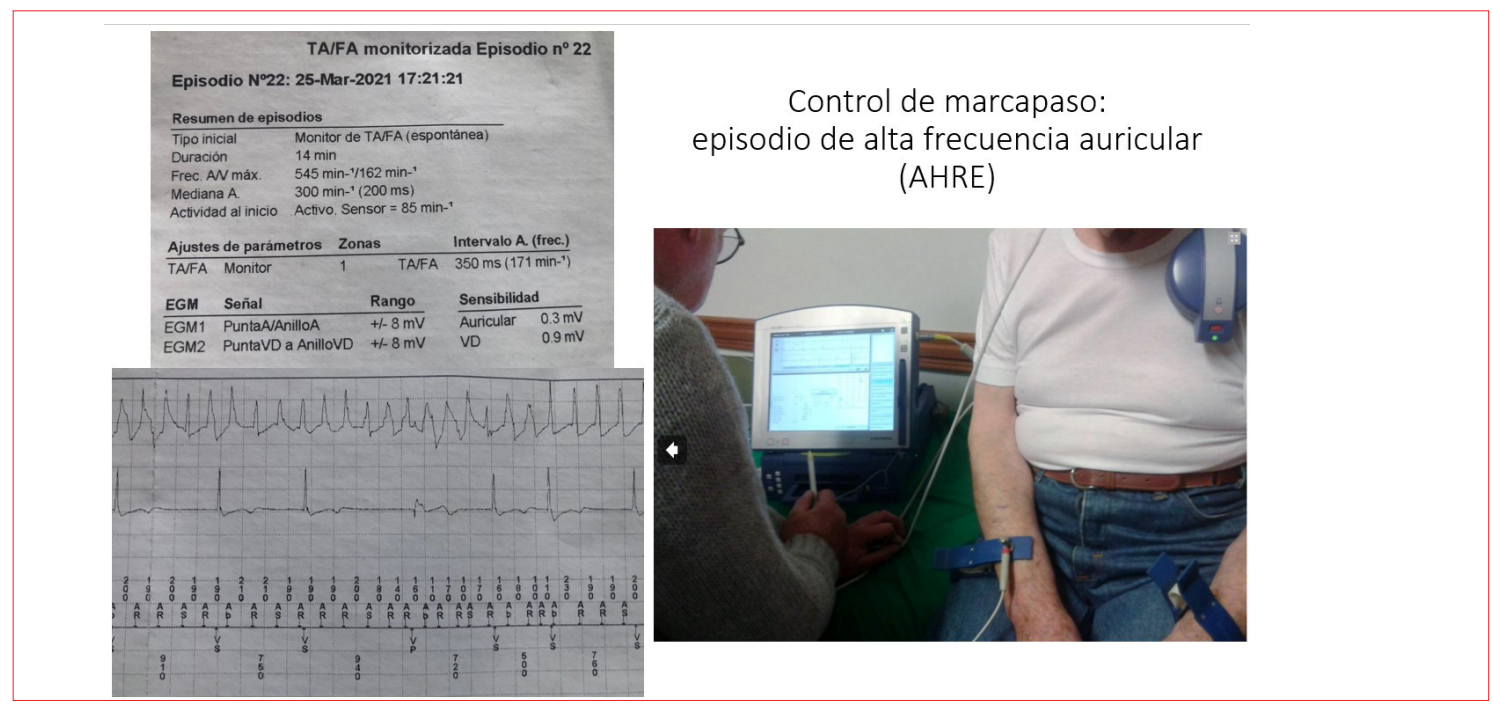

Figura 8. Registros tomados del programador durante el control rutinario de un marcapaso bicameral en policlínica. Arriba: detalles del episodio, fecha, hora, duración. Debajo: registro intracavitario sensado a través de los electrodos auricular y ventricular; de arriba abajo, registro de actividad auricular, registro de actividad ventricular, canal con medida de los intervalos auriculares y ventriculares. AR: registro auricular durante el período refractario; AS: sensado de actividad auricular; VS: sensado de actividad ventricular; VP: actividad ventricular estimulada.

grupo aproximadamente). El período de estudio fue de un año, y en ambos grupos de intervención se identificaron más casos nuevos de FA comparativamente con la conducta habitual (OR 1,6; 95\% IC 1,1-2,3) ${ }^{(30)}$. Como fue también demostrado, el tiempo de monitorización aumenta de manera significativa la detección de $\mathrm{FA}^{(18,22)}$. De forma concordante con el aumento de la prevalencia de FA y de ACV, el rendimiento de las estrategias de screening aumenta con la edad, no se indica en pacientes asintomáticos menores de 65 años si no hay factores de riesgo que lo justifiquen, por ejemplo, antecedentes de hipertensión arterial o de apnea obstructiva del sueño. Recientemente el grupo de trabajo de la ESC en Cardiología Digital $(e \text {-Cardiology })^{(4)}$ estableció recomendaciones referidas a las estrategias para el tamizaje de FA, que resumimos en la tabla 3 .

A nivel global, más del $80 \%$ de los $\mathrm{ACV}$ son isquémicos y de ellos en un 15\%-40\% no se puede definir una causa y de manera genérica se denominan criptogénicos. El grupo de pacientes que presenta un ACV embólico en los que no se detecta una causa clara ni se registra FA por los métodos tradicionales (ESUS: Embolic Stroke of Undetermined Source), tiene un elevado riesgo de recu- 


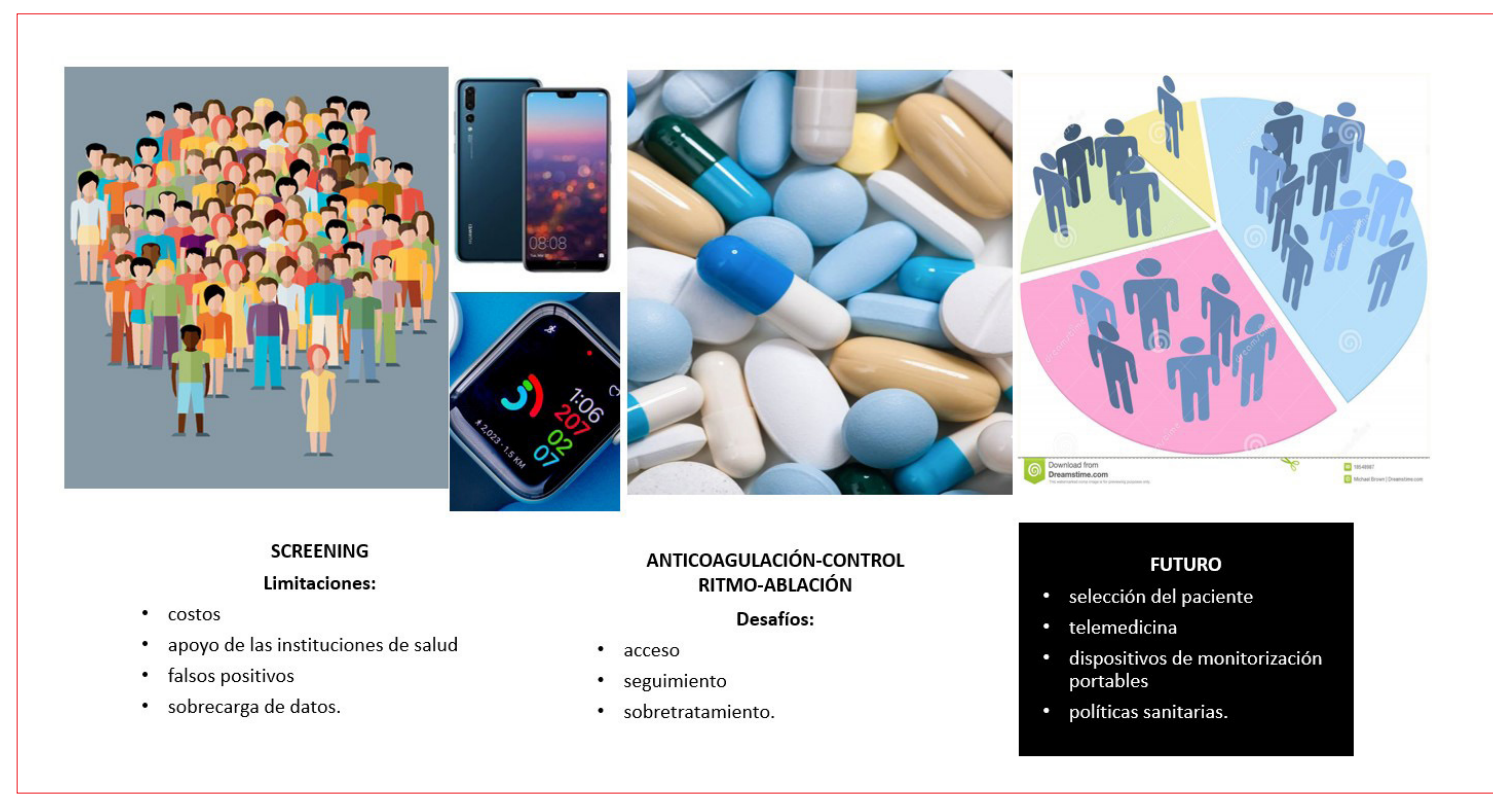

Figura 9. Situación actual, desafíos y futuro de la detección de la FA.

rrencias y de lesiones isquémicas clínicamente silenciosas. La mejor estrategia para la prevención secundaria en este grupo significativo de pacientes genera siempre un problema clínico: ¿antiagregación o anticoagulación? Dos estudios de reciente publicación valoraron en esta población: uno de ellos, el uso de rivaroxaban $15 \mathrm{mg}$ versus aspirina $100 \mathrm{mg}^{(31)}$; y el otro, dabigatrán 150/110 mg x2 versus aspirina $100 \mathrm{mg}^{(32)}$. En ninguno de los dos estudios los anticoagulantes directos usados empíricamente mostraron superioridad sobre el uso de aspirina y sí una mayor incidencia de sangrados en el caso del rivaroxaban. Estos resultados jerarquizan aún más la importancia de profundizar la metodología para detección de FA. El monitoreo prolongado permitirá definir con mayor certeza la necesidad del uso de anticoagulantes y evitar su empleo en forma empírica ${ }^{(9)}$.

El ensayo EMBRACE (Event Monitor Belt for Recording Atrial fibrillation after a Cerebral Isquemic Event) incluyó 572 pacientes y comparó el monitoreo durante 30 días con un registrador de eventos versus Holter de 24 horas realizado dentro de los 90 días del episodio isquémico cerebral. Se detectó $16 \%$ de FA en el primer grupo versus $3,2 \%$ en el grupo Holter ( $p<0,001)$; el número de pacientes a monitorizar por cada FA detectada fue de $8^{(33)}$. Por otra parte, el estudio CRYSTAL-AF (Cryptogenic Stroke and Underlying Atrial Fibrillation) en una población pos-ACV isquémico sin FA documentada en ECG o Holter de 24 horas, comparó el uso de un dispositivo implantable (n: 221) versus los controles habituales (n: 220). El grupo implantado mostró una frecuencia de detección de FA 6 veces mayor a los 6 meses, que alcanzó al 30\% del grupo a los 3 años ${ }^{(34)}$. El monitoreo prolongado se traduce en una reducción del 51\% en el riesgo de recurrencia de ACV de acuerdo con un reciente metaanálisis. Este análisis que incluyó 28 estudios con un total de 4.531 pacientes mostró que la detección de FA luego de un evento isquémico cerebral se asoció solo con dos factores: la edad y la duración de la monitorización ${ }^{(35)}$.

En pacientes portadores de marcapasos o cardiodesfibriladores que permiten el registro de la actividad auricular (catéter atrial o electrodos proximales para sensado en electrodo ventricular) se pueden detectar episodios de frecuencia rápida auricular, Atrial High Rate Episodes (AHRE, por su sigla en inglés). Integran el espectro de la llamada FA subclínica, aunque a veces se utilizan estos términos como sinónimos. Si bien no existe un límite específico de frecuencia, habitualmente los dispositivos lo definen por defecto como una frecuencia $\geq 175 \mathrm{pm}$. Debido a su intermitencia y a veces corta duración, es casi imposible detectarlos por métodos de registro externos. Constituye una recomendación clase I en las guías de FA de la ESC 2020(6) que al controlar periódicamente marcapasos y cardiodesfibriladores se revisen los electrogramas auriculares almacenados y se confirme si se trata de AHRE, valorando su incidencia general, duración, aspectos que además ayudan a determinar la "carga" de FA (figura 8).

Es habitual que la población portadora de marcapasos sea añosa, por tanto, el registro de 


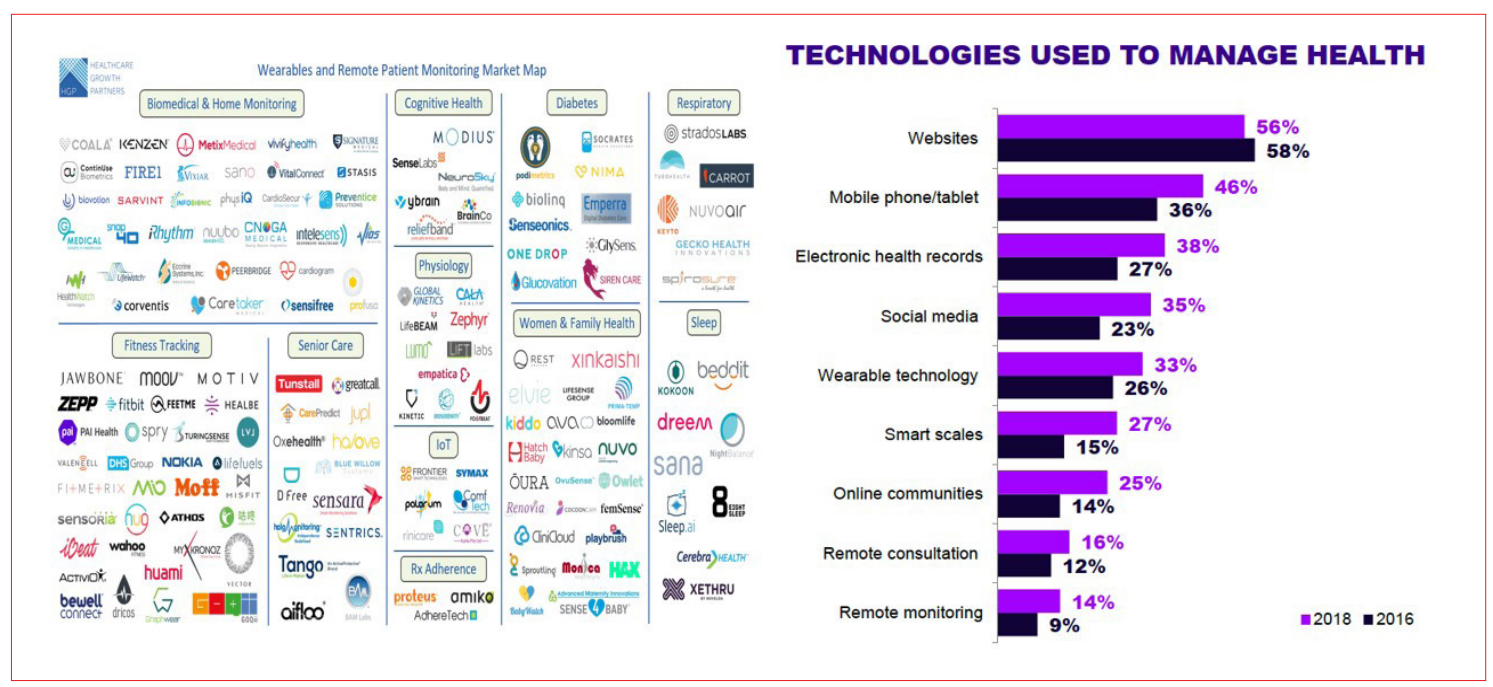

Figura 10. Derecha: evolución 2016-2018 del uso por la población de los recursos tecnológicos para el cuidado de la salud. Izquierda: empresas tecnológicas dedicadas al desarrollo de aplicaciones o dispositivos. De referencias 44 y 45.

AHRE en pacientes con dispositivos es frecuente, estimándose entre un $30 \%-70 \%{ }^{(36)}$. Los episodios breves, de segundos de duración, son irrelevantes desde el punto de vista clínico. Sin embargo, cuando son prolongados se asocian a mayor riesgo de FA, ACV isquémico y eventos cardiovasculares mayores. Se ha reportado además que 1 de cada 5-6 pacientes con AHRE desarrollarán FA en un período de 2,5 años ${ }^{(37)}$. Sin embargo, todavía no está claro el riesgo de ACV de esta población en comparación con una población con FA detectada clínicamente. La indicación de tratamiento anticoagulante es, entonces, todavía motivo de debate, debiendo considerarse siempre el riesgo global del paciente. La evidencia actual sugiere considerar su uso cuando los episodios duran más de 24 horas y es obligatorio cuando el score $\mathrm{CHA}_{2} \mathrm{DS}_{2}$ VASc es $\geq 2$. Puede estar justificado también cuando, si bien la duración es menor, el riesgo es elevado. En cualquier caso, estos pacientes requieren de un control más estricto, monitorización regular y reevaluación periódica del score de riesgo. Existe en curso una serie de ensayos clínicos que aportarán más información sobre ese punto.

Más allá del diagnóstico de fibrilación auricular: el rol de la tecnología en el manejo y la prevención

Las herramientas digitales disponibles también facilitan al clínico el manejo y el seguimiento de los pacientes con FA ya conocida. Agregado a su aporte en el diagnóstico inicial, su utilidad es fácilmente comprensible en la valoración del mantenimiento del ritmo sinusal luego de una cardio- versión o de una ablación, por ejemplo. Además, permiten valorar y controlar otros aspectos que, como en la mayoría de las enfermedades crónicas no trasmisibles, son muy difíciles de seguir en forma continua y objetiva en la práctica asistencial tradicional. Entre ellos la adherencia al tratamiento, los cambios de estilo de vida, el autocontrol, pudiendo aportar también aspectos educativos fundamentales. Está demostrado que el mayor empoderamiento de los pacientes respecto a su patología y su control mejora los resultados clínicos.

El ensayo $m A F$-App Trial reporta la experiencia con el uso de una aplicación sencilla que incorpora datos clínicos y antecedentes del paciente, los scores de riesgo de ACV y sangrado, además de material educativo, diferentes estrategias para involucrar al paciente y protocolos de autocuidado y seguimiento ${ }^{(38)}$. En el piloto se randomizaron 113 pacientes para el uso de la app y 96 para el control habitual. El uso de la app se tradujo en una mejoría en el conocimiento de la patología, en la adherencia al tratamiento, sobre todo la aceptación del tratamiento anticoagulante y en la calidad de vida. El mismo grupo de investigadores (mAF-App II Trial Investigators) publicó recientemente un ensayo ${ }^{(39)}$ a mayor escala realizado en 40 ciudades de China, con el objetivo de demostrar si el uso de la tecnología móvil como parte de una estrategia de manejo del paciente con FA (1.646 pacientes) reducía los eventos adversos en relación con el control habitual (1.678 pacientes). Con el apoyo tecnológico, el punto final combinado de ACV + tromboembolismo, rehospitalizaciones $\mathrm{y}$ muerte fue significativamente menor (1,9\% ver- 
sus $6,0 \%$, HR 0,39; 95\% IC 0,22-0,67; p < 0,001).

$\mathrm{Al}$ inicio de la pandemia por coronavirus la clínica de FA del Centro Médico de la Universidad de Maastricht canceló las consultas presenciales de los pacientes con FA que pasaron a realizarse en formato de teleconsulta. En apoyo a esta modalidad de relación con el paciente se instrumentaron recursos de mSalud para monitorización del ritmo y la frecuencia cardíaca usando apps, proyecto denominado TeleCheck-AF, que se fundamentó en tres pilares: la teleconsulta, el control del ritmo y frecuencia y el enfoque multidisciplinario $^{(40)}$ (tabla 4).

El protocolo TeleCheck-AF es aplicado en numerosos centros europeos. Recientemente los investigadores presentaron en el Congreso de la EHRA 2021 los resultados de dos encuestas realizadas a los centros participantes, una sobre las características del centro (25 sitios) y otra sobre la experiencia en sí misma (23 sitios). Se reclutaron 1.930 pacientes en 28 semanas. La mayoría de los centros eran académicos y tenían clínicas de FA especializadas (64\%). La experiencia reportada fue ampliamente satisfactoria: uso sencillo y accesible tanto para el médico como para los pacientes, aceptación y confiabilidad de los datos. En otro reporte se resume una encuesta de satisfacción realizada a los pacientes (completada por 826 pacientes). La aceptación fue ampliamente mayoritaria, con la mayor adherencia lograda en la población de más de 80 años y el deseo de mantener esta alternativa luego de la pandemia ${ }^{(41,42)}$.

La Inteligencia Artificial (IA) se utiliza en electrofisiología desde hace décadas y es aplicada para el análisis de los registros obtenidos mediante el uso de PPG en teléfonos celulares y relojes inteligentes. En la actualidad, sumado al desarrollo tecnológico en sensores, al mayor poder de las computadoras y a la existencia de plataformas basadas en la web, sus usos se han potenciado. El avance en las telecomunicaciones, el wifi, los celulares inteligentes, junto a conceptos nuevos como el de internet de las cosas, que refiere a la interconexión digital de objetos cotidianos con internet, han permitido, como vimos, el monitoreo de grandes poblaciones para la detección de FA, un hecho que hasta hace no muchos años era inimaginable. Este desarrollo seguramente contribuirá en un futuro cercano, además del diagnóstico y el seguimiento de la FA, a su prevención. Un modelo de IA fue aplicado para el análisis del ECG basal en ritmo sinusal, utilizando una muestra de $10^{\prime \prime}$ de las 12 derivaciones de registros de la base de datos de la Clínica Mayo de Estados Unidos ${ }^{(43)}$. Se analizaron 649.931 ECG correspondientes a 180.922 pacientes. Se logró identificar a los pacientes que tendrían FA por el ECG en ritmo sinusal con una sensibilidad y especificidad del $79 \%$. Si se consideraban los ECG registrados en el mes previo a la primera FA, la sensibilidad fue del 82,3\% y la especificidad de 83,4\% (área bajo la curva: 0,90).

\section{Lo que falta}

Más allá de la información resumida, vinculada específicamente a la FA, quedan aún muchas interrogantes que sin duda tendrán respuesta en los próximos años. A modo de ejemplos: ¿cuál es el número de personas a controlar para prevenir un ACV?, ¿cuánto tiempo debería monitorizarse una persona de riesgo?, ¿cuáles son las estrategias de detección de mayor rendimiento?, o ¿cuánto debe durar el screening luego de un ACV? Además, las nuevas tecnologías tendrán que ser validadas en ensayos clínicos, deberán incorporarse a la historia clínica electrónica y también permitir el diagnóstico de otras arritmias (figura 9).

Por último, pero muy importante, la indicación y los resultados deben ser analizados en el contexto global e individual de cada paciente.

A pesar de estas limitaciones, el uso y la aceptación de los recursos tecnológicos en los cuidados de salud está cada vez más difundido entre los pacientes/consumidores. Sumado a la investigación y al desarrollo de diferentes tipos de dispositivos para monitorizar los parámetros biológicos por empresas tecnológicas, inevitablemente estos procesos impulsarán su aplicación en la rutina asistencial $^{(44,45)}$ (figura 10).

\section{Conclusiones}

El concepto de salud digital comprende el uso de tecnologías de la comunicación que permiten colectar y analizar información digital para mejorar la salud del paciente, su educación y asegurarle una correcta asistencia. Involucra a la telesalud, registros electrónicos, dispositivos de monitorización implantables o sensores portátiles, IA, ciencias del comportamiento y a la llamada medicina personalizada. La mSalud es parte de este concepto. Quizás los especialistas más adaptados a su uso sean los electrofisiólogos, pero el desarrollo de los sensores hará que esta modalidad asistencial se expanda a todas las especialidades, principalmente a aquellas que manejan patologías crónicas no trasmisibles.

La mSalud se encuentra en diferentes etapas de desarrollo en el mundo. Solo algunas de las tecnologías resumidas en este artículo están apro- 
badas a escala global o son financiadas en todos los países. La OMS ha planteado que el aumento de la capacidad de implementar y desarrollar a gran escala estrategias innovadoras y rentables en salud digital jugará un papel fundamental para conseguir el objetivo de una cobertura sanitaria universal, al mismo tiempo que aseguraría servicios de calidad $^{(3)}$. Para cumplir este objetivo deben superarse algunas barreras, sin embargo, la pandemia por coronavirus permitió visualizar que la base tecnológica existe y que muchos obstáculos pueden resolverse con facilidad. La mSalud revolucionará la forma de interacción entre las poblaciones y los servicios de salud en el mundo.

Finalmente, en referencia concreta al screening de FA y más allá de la enorme ayuda que el desarrollo y uso de la tecnología aportan en la práctica, su indicación y sus resultados deben ser siempre analizados en el contexto global e individual de cada paciente y de acuerdo con la evidencia científica.

El uso de la tecnología digital en salud, que incluye la genómica, la medicina digital, la inteligencia artificial y la robótica, no debe ser visto simplemente como un aumento en los costos. Se trata de una nueva manera de abordar los enormes desafíos en los cuidados de la salud del siglo XXI. The Topol Review. Preparing the healthcare work force to deliver the digital future. NHS (National Health Service). Reino Unido. Febrero, 2019.

Walter Reyes Caorsi, ORCID 0000-0002-4670-5765.

Este artículo fue aprobado para su publicación por el Editor adjunto, Dr. Ignacio Farro

\section{Bibliografía}

1. World Health Organization. mHealth: new horizons for health through mobile technologies. Based on the findings of the second global survey on health. Geneva: WHO, 2011. Disponible en: https://www. who.int/goe/publications/goe_mhealth_web.pdf. [Consulta: 14 Oct 2021]

2. Dagher L, Marrouche N. Consumer-tech-provider co-doctoring in the digital age: a neglected TRIAD. Heart Rhythm 2021; 18(4):499-500. doi: 10.1016/j. hrthm.2020.10.007

3. World Health Organization. WHO guideline: recommendations on digital interventions for health system strengthening. Geneva: WHO, 2019. Disponible en: https://www.who.int/publications/i/ item/9789241550505. [Consulta: 14 Oct 2021].

4. Jensen M, Treskers R, Caiani E, Casado-Arroyo R, Cowie M, Dilaveris P, et al. ESC working group in e-cardiology position paper: use of commercially available wearable technology for heart rate and activity tracking in primary and secondary cardiovascular prevention-in collaboration with the European Heart Rhythm Association, European Association of Preventive Cardiology, Association of Cardiovascular Nursing and Allied Professionals, Patient Forum, and the Digital Health Committee. Eur Heart J Digit Health 2021; 2(1):49-59. doi: 10.1093/ehjdh/ztab011

5. Wan E, Ghanbari H, Akoum N, Attia Z, Asirvatham S, Chung E, et al. HRS White Paper on clinical utilization of digital health technology. Cardiovasc Digit Health J 2021; 2(4):196-211. doi: 10.1016/j. cvdhj.2021.07.001

6. Hindricks G, Potpara T, Dagres N, Arbelo E, Bax J, Blomström-Lundqvist C, et al; ESC Scientific Document Group. 2020 ESC Guidelines for the diagnosis and management of atrial fibrillation developed in collaboration with the European Association for Cardio-Thoracic Surgery (EACTS): The Task Force for the diagnosis and management of atrial fibrillation of the European Society of Cardiology (ESC) Developed with the special contribution of the European Heart Rhythm Association (EHRA) of the ESC. Eur Heart J 2021; 42(5):373-498. doi: 10.1093/ eurheartj/ehaa612

7. Andrade J, Aguilar M, Atzema C, Bell A, Cairns $\mathrm{J}$, Cheung C, et al; Members of the Secondary Panel. The 2020 Canadian Cardiovascular Society/ Canadian Heart Rhythm Society comprehensive guidelines for the management of atrial fibrillation. Can J Cardiol 2020; 36(12):1847-948. doi: 10.1016/j. cjca.2020.09.001

8. Mairesse G, Moran P, Van Gelder I, Elsner C, Rosenqvist M, Mant J, et al; ESC Scientific Document Group. Screening for atrial fibrillation: a European Heart Rhythm Association (EHRA) consensus document endorsed by the Heart Rhythm Society (HRS), Asia Pacific Heart Rhythm Society (APHRS), and Sociedad Latinoamericana de Estimulación Cardíaca y Electrofisiología (SOLAECE). Europace 2017; 19(10):1589-623. doi: 10.1093/europace/eux177

9. Varma N, Cygankiewicz I, Turakhia M, Heidbuchel H, Hu Y, Chen L, et al. 2021 Position Paper ISHNE/HRS/EHRA/APHRS collaborative statement on mHealth in arrhythmia management: digital medical tools for heart rhythm professionals. Cardiovasc Digit Health J 2021; 2(1):4-54. doi: 10.1016/j.cvdhj.2020.11.004

10. Reyes Caorsi W. Efectos colaterales positivos de la pandemia (II): introducción a la e-Salud. Rev Urug Cardiol 2020; 35(3):276-84. Doi: 10.29277/cardio.35.3.5

11. RockHealth. Stanford Center for Digital Health. Digital Health Consumer Adoption Report 2020. Stanford, CA: Stanford Center for Digital Health, 2020. Disponible en: https://rockhealth.com/reports/digital-health-consumer-adoption-report-2020/. [Consulta:].

12. Ding E, Svennberg E, Wurster C, Duncker D, Manninger M, Lubitz S, et al. Survey of current 
perspectives on consumer-available digital health devices for detecting atrial fibrillation. Cardiovasc Digit Health J 2020; 1(1):21-9. doi: 10.1016/j.cvdhj.2020.06.002

13. Manninger M, Kosiuk J, Zweiker D, Njeim M, Antolic B, Kircanski B, et al. Role of wearable rhythm recordings in clinical decision making-The wEHRAbles project. Clin Cardiol 2020; 43(9):1032-9. doi: 10.1002/clc. 23404

14. Avezum A, Cantú C, González-Zuelgaray J, True Hills M, Lobban T, Massaro A, et al. ¿Cómo prevenir los accidentes cerebrovasculares en Latinoamérica? Informe del grupo de trabajo: Prevención de accidentes crebrovasculares en pacientes con fibrilación auricular. Action for Stroke Prevention, 2011. Disponible en: https://www.stopafib.org/ downloads/News362.pdf. [Consulta:].

15. Friberg L, Rosenqvist M, Lindgren A, Terént A, Norrving B, Asplund K. High prevalence of atrial fibrillation among patients with ischemic stroke. Stroke 2014; 45(9):2599-605. doi: 10.1161/STROKEAHA. 114.006070

16. Diener H, Hart R, Koudstaal P, Lane D, Lip G. Atrial fibrillation and cognitive function: JACC review topic of the week. J Am Coll Cardiol 2019; 73(5):612-9. doi: 10.1016/j.jacc.2018.10.077

17. Freedman B, Camm J, Calkins H, Healey J, Rosenqvist M, Wang J, et al; AF-Screen Collaborators. Screening for atrial fibrillation: a report of the AFSCREEN International Collaboration. Circulation 2017; 135(19):1851-67. doi: 10.1161/CIRCULATIONAHA.116.026693

18. Svennberg E. Benefits of systematic screening for atrial fibrillation: the STROKESTOP-study. Late Breaking Clinical Trials Session. In: EHRA 2021, April 23-25 2021. Disponible en: https://esc365.escardio.org/presentation/232051. [Consulta:].

19. Svennberg E, Friberg L, Frykman V, Al-Khalili F, Engdahl J, Rosenqvist M. Clinical outcomes in systematic screening for atrial fibrillation (STROKESTOP): a multicentre, parallel group, unmasked, randomised controlled trial. Lancet 2021. doi: 10.1016/S0140-6736(21)01637-8. [Artículo en pren$\mathrm{sa}$.

20. Engler D, Heidbuchel H, Schnabel R. Digital, risk-based screening for atrial fibrillation in the European community-the AFFECT-EU project funded by the European Union. Eur Heart J 2021; 42(27):2625-7. doi: 10.1093/eurheartj/ehab050

21. Zungsontiporn N, Link M. Newer technologies for detection of atrial fibrillation. BMJ 2018; 363:k3946. doi: 10.1136/bmj.k3946

22. Halcox J, Wareham K, Cardew A, Gilmore M, Barry J, Phillips C, et al. Assessment of remote heart rhythm sampling using the alivecor heart monitor to screen for atrial fibrillation: the REHEARSE-AF study. Circulation 2017; 136(19):1784-94. doi: 10.1161/CIRCULATIONAHA.117.030583

23. Estrago V, Muñoz M, Álvarez R, Reyes X, Reyes W. Utilización de un dispositivo móvil de tecnología electrónica para tamizaje de fibrilación auricular: estudio piloto. Rev Urug Cardiol 2021; 36(2):e201. doi: 10.29277/cardio.36.2.7

24. Zhang W, Chen Y, Miao C, Huang Q, Sheng C, Shao $\mathrm{S}$, et al. Quarterly versus annual ECG screening for atrial fibrillation in older Chinese individuals (AF-CATCH): a prospective, randomized controlled trial. Lancet Healthy Longev 2021; 2:e470-8. doi: 10.1016/S2666-7568(21)00138-0

25. Perez M, Mahaffey K, Hedlin H, Rumsfeld J, Garcia A, Ferris T, et al; Apple Heart Study Investigators. Large-scale assessment of a smartwatch to identify atrial fibrillation. N Engl J Med 2019; 381(20):190917. doi: 10.1056/NEJMoa1901183

26. Guo Y, Wang H, Zhang H, Liu T, Liang Z, Xia Y, et al; MAFA II Investigators. Mobile Photoplethysmographic Technology to Detect Atrial Fibrillation. J Am Coll Cardiol 2019; 74(19):2365-75. doi: 10.1016/j.jacc.2019.08.019

27. Yan B, Lai W, Chan C, Chan S, Chan L, Lam K, et al. Contact-Free screening of atrial fibrillation by a smartphone using facial pulsatile photoplethysmographic signals. J Am Heart Assoc 2018; 7(8):e008585. doi: 10.1161/JAHA.118.008585

28. Wang A, Nguyen D, Sridhar A, Gollakota S. Using smart speakers to contactlessly monitor heart rhythms. Commun Biol 2021; 4(1):319. doi: 10.1038/ s42003-021-01824-9

29. Lowres N, Olivier J, Chao T, Chen S, Chen Y, Diederichsen A, et al. Estimated stroke risk, yield, and number needed to screen for atrial fibrillation detected through single time screening: a multicountry patient-level meta-analysis of 141,220 screened individuals. PLoS Med 2019; 16(9):e1002903. doi: 10.1371/journal.pmed.1002903

30. Hobbs F, Fitzmaurice D, Mant J, Murray E, Jowett $\mathrm{S}$, Bryan $\mathrm{S}$, et al. A randomised controlled trial and cost-effectiveness study of systematic screening (targeted and total population screening) versus routine practice for the detection of atrial fibrillation in people aged 65 and over. The SAFE study. Health Technol Assess 2005; 9(40):1-74. doi: 10.3310/hta9400

31. Hart R, Sharma M, Mundl H, Kasner S, Bangdiwala S, Berkowitz S, et al; NAVIGATE ESUS Investigators. Rivaroxaban for stroke prevention after embolic stroke of undetermined source. N Engl J Med 2018; 378(23):2191-201. doi: 10.1056/NEJMoa1802686

32. Diener H, Sacco R, Easton J, Granger C, Bernstein R, Uchiyama S, et al; RE-SPECT ESUS Steering Committee and Investigators. Dabigatran for prevention of stroke after embolic stroke of undetermined source. N Engl J Med 2019; 380(20):1906-17. doi: 10.1056/NEJMoa1813959

33. Gladstone D, Spring M, Dorian P, Panzov V, Thorpe $\mathrm{K}$, Hall J, et al; EMBRACE Investigators and Coordinators. Atrial fibrillation in patients with cryptogenic stroke. N Engl J Med 2014; 370(26):2467-77. doi: 10.1056/NEJMoa1311376

34. Sanna T, Diener H, Passman R, Di Lazzaro V, Bernstein R, Morillo C, et al; CRYSTAL AF Investiga- 
tors. Cryptogenic stroke and underlying atrial fibrillation. N Engl J Med 2014; 370(26):2478-86. doi: 10.1056/NEJMoa1313600

35. Tsivgoulis G, Katsanos A, Köhrmann M, Caso V, Perren F, Palaiodimou L, et al. Duration of implantable cardiac monitoring and detection of atrial fibrillation in ischemic stroke patients: a systematic review and meta-analysis. J Stroke 2019; 21(3):30211. doi: $10.5853 /$ jos.2019.01067

36. Diederichsen S, Haugan K, Brandes A, Lanng M, Graff C, Krieger D, et al. Natural history of subclinical atrial fibrillation detected by implanted loop recorders. J Am Coll Cardiol 2019; 74(22):2771-81. doi: 10.1016/j.jacc.2019.09.050

37. Healey J, Connolly S, Gold M, Israel C, Van Gelder I, Capucci A, et al; ASSERT Investigators. Subclinical atrial fibrillation and the risk of stroke. $\mathrm{N}$ Engl J Med 2012; 366(2):120-9. doi: 10.1056/NEJMoa1105575

38. Guo Y, Chen Y, Lane D, Liu L, Wang Y, Lip G. Mobile health technology for atrial fibrillation management integrating decision support, education, and patient involvement: maf app trial. Am J Med 2017; 130(12):1388-96.e6. doi: 10.1016/j.amjmed.2017.07.003

39. Guo Y, Lane D, Wang L, Zhang H, Wang H, Zhang $\mathrm{W}$, et al; mAF-App II Trial Investigators. Mobile health technology to improve care for patients with atrial fibrillation. J Am Coll Cardiol 2020; 75(13):1523-34. doi: 10.1016/j.jacc.2020.01.052

40. Pluymaekers N, Hermans A, van der Velden R, Gawałko M, den Uijl D, Buskes S, et al. Implementation of an on-demand app-based heart rate and rhythm monitoring infrastructure for the management of atrial fibrillation through teleconsultation:
TeleCheck-AF. Europace 2021; 23(3):345-52. doi: 10.1093/europace/euaa201

41. Linz D, Pluymaekers N, Hermans A, Van Der Velden R, Verhaert D, Gupta D, et al. Remote app-based management of atrial fibrillation during the COVID-19: the centre characteristics and experiences of the European TeleCheck-AF project. Europace 2021; 23(Suppl 3):iii574. doi: 10.1093/europace/ euab116.522

42. Linz D, Pluymaekers N, Van Der Velden R, Hermans A, Verhaert D, Hemels M, et al. The TeleCheck-AF project on remote app-based management of atrial fibrillation during the COVID-19 pandemic: Patient experiences. Europace 2021; 23(Suppl 3):iii573. doi: 10.1093/europace/euab116.521

43. Attia Z, Noseworthy P, Lopez-Jimenez F, Asirvatham S, Deshmukh A, Gersh B, et al. An artificial intelligence-enabled ECG algorithm for the identification of patients with atrial fibrillation during sinus rhythm: a retrospective analysis of outcome prediction. Lancet 2019; 394(10201):861-7. doi: 10.1016/S0140-6736(19)31721-0

44. Accenture Consulting. 2018 consumer survey on digital health: US results. Disponible en: https:// www.accenture.com/t20180306t103559z_w__/usen/_acnmedia/pdf-71/accenture-health-2018-consumer-survey-digital-health.pdf. [Consulta: 14 Oct 2021].

45. Healthcare Growth Partners. Reaching the healthcare mainstream: wearables and remote patient monitoring market map. Atlanta, GA: Hit Consultant Media, 2019. Disponible en: https://hitconsultant.net/2019/06/21/wearables-and-remote-patient-monitoring-market-map/\#.YWc5kCHMLcc. [Consulta: 14 Oct 2021]. 